1 eSPRESSO: a spatial self-organizing-map clustering method for

2 single-cell transcriptomes of various tissue structures using

3 graph-based networks

4

5

6 Authors

7 Tomoya Mori ${ }^{1}$, Toshiro Takase ${ }^{2}$, Kuan-Chun Lan $^{3}$, Junko Yamane ${ }^{3}$, Cantas Alev ${ }^{4}$, Kenji Osafune ${ }^{3}$,

8 Jun Yamashisa ${ }^{3}$, and Wataru Fujibuchi ${ }^{3, *}$

9

10 Affiliations

$11{ }^{1}$ Bioinformatics Center, Institute for Chemical Research, Kyoto University, Gokasho, Uji, Kyoto

12 611-0011, Japan.

132 Life Science, Global Business Services, IBM Japan Ltd, 19-21 Nihonbashi Hakozaki-cho, Chuo-ku,

14 Tokyo 103-8510, Japan

$15{ }^{3}$ Center for iPS Cell Research and Application (CiRA), Kyoto University, 53 Kawahara-cho,

16 Sho-goin, Sakyo-ku, Kyoto 606-8507, Japan

$17{ }^{4}$ Institute for the Advanced Study of Human Biology (ASHBi), Kyoto University, Medical Faculty

18 Bldg. B, Alev-Lab, Yoshida-Konoe-cho, Sakyo-ku, Kyoto 606-8501, Japan 


\section{Telephones and e-mail addresses}

21 Tomoya Mori: +81-774-38-3016. E-mail: tmori@ kuicr.kyoto-u.ac.jp

22 Toshiro Takase: +81-80-5915-4235, e30809@jp.ibm.com

23 Kuan-Chun Lan: +81-75-366-7014, k.lan@cira.kyoto-u.ac.jp

24 Junko Yamane: +81-75-366-7184, yamane-j@cira.kyoto-u.ac.jp

25 Cantas Alev: +81-75-753-9899, alev.cantas.8m@kyoto-u.ac.jp

26 Kenji Osafune: +81-75-366-7058, osafu@ cira.kyoto-u.ac.jp

27 Jun Yamashita: +81-75-366-7057, juny@ cira.kyoto-u.ac.jp

28 Wataru Fujibuchi* : +81-75-366-7012, fujibuchi-g@ cira.kyoto-u.ac.jp

$29 *$ Corresponding author

30 


\section{ABSTRACT}

32 Animal cells are spatially organized as tissues and cellular gene expression data contain such

33 information that governs body structure and morphogenesis during developmental process. Although

34 several computational tissue reconstruction methods using transcriptomic data have been proposed,

35 those methods are insufficient with regard to arranging cells in their correct positions in tissues or

36 organs unless spatial information is explicitly provided. Here, we propose eSPRESSO, a powerful in

37 silico three-dimensional (3D) tissue reconstruction method using stochastic self-organizing map

38 (stochastic-SOM) clustering, together with optimization of gene set by Markov chain Monte Carlo

39 (MCMC) framework, to estimate the spatial domain structure of cells in any topology of tissues or

40 organs from only their transcriptome profiles. We confirmed the performance of eSPRESSO by

41 mouse embryo, embryonic heart, adult cortical layers, and human pancreas organoid with high

42 reproducibility ( success rate $=72.5-100 \%$ ), while discovering morphologically important spatial

43 discriminator genes (SDGs). Furthermore, we applied eSPRESSO to analysis of human adult heart

44 diseases by virtual gene knockouts, and revealed candidate mechanisms of deformation of heart

45 structure. The eSPRESSO may provide novel methods to analyze the mechanisms of 3D structure

formation and morphology-based disease mechanisms. 


\section{INTRODUCTION}

48 Analysis of disease mechanisms based on high-throughput single-cell RNA-sequencing is becoming

49 prominent and fundamental technique in cell biology ${ }^{1-3}$. Particularly, methods for high-throughput,

50 spatially resolved single-cell RNA-seq have been developed and attracting attentions as novel

51 analytics for disease mechanisms. Single-molecule fluorescence in-situ hybridization (FISH) ${ }^{4}$ has

52 been widely used to quantitate transcript numbers at single-cell resolution, often within the context

53 of a diseased tissues of interest. More recently, highly-multiplexed methods such as seqFISH ${ }^{5}$ or

54 merFISH $^{6}$ are reported to detect transcript dynamics of target cells in 3D location. More directly,

55 high-resolved, 2D-mapped primer-based RNA-sequencing of tissues fixed on a glass plate has been

56 also developed for mapping of transcript abundance, utilized to draw spatial cellular location map

57 after reconstructing 3D image by multiple 2D-maps ${ }^{7}$. However, these methods are still in the early

58 stage and requires further research and development in terms of practical cost and conveniences.

Alternatively, several computational methods to reconstruct 3D tissues by estimating the

60 spatial positions of individual cells in tissues with gene expression data obtained by single-cell

61 RNA-seq have been reported. ${ }^{8-14}$ These methods may be roughly divided into two types: the

62 landmark approach and the $a b$ initio approach. The landmark approach estimates the 3D position of

63 each cell based on gene expression profiles while using the spatial information of marker genes

64 obtained by in situ hybridization..$^{8-10}$ Conversely, the ab initio approach assigns each cell to 3D space 
65 according to the principal component score calculated from gene expression profiles without using

66 such spatial reference data. ${ }^{11-14}$ Although current principal component analysis (PCA)-based

67 methods are simply used for 3D visualization, an ab initio approach that does not depend on the spatial information of marker genes obtained by in situ hybridization is promising for 3D reconstruction. Previously, we reported a novel 3D reconstruction method using stochastic-SOM

71 clustering, or SPRESSO (SPatial REconstruction by Stochastic-SOM) ${ }^{15}$, which features gene

72 selections based on Gene Ontology (GO). ${ }^{16,17}$ The method yielded high success rates of 3D

73 reconstructions of mouse gastrula embryo and demonstrated a remarkable ability to find SDGs that

74 contribute to differentiation and tissue morphogenesis. This method, however, was preliminary and simply projected four domains of mouse gastrula to a cubic structure and thus unapplicable to more complicated structures of organs such as heart or pancreas. domains or tissues as long as they can be drawn as some kind of connection graphs or network

79 diagrams. The concept of graph-based SOM was already reported in 1989 by T. Kohonen and

80 colleagues $^{18}$ who introduced a Kohonen map or network in $1982^{19}$, which is one of the artificial

81 neural networks and computationally convenient abstraction building on biological models of

82 neuronal systems ${ }^{20}$ and morphogenesis models by Alan Turing ${ }^{21}$. So far, many useful topology 
83 structures other than square grids such as toroidal grids that have opposite edges are connected ${ }^{22}$

84 have been introduced. However, graph-network representation is the most simple but comprehensive

85 for describing relationships tissue domains. Thus, we applied graph-SOM to various types of mouse

86 and human organs that show specific topologies of tissue domain relationships. We also successfully

87 visualized the reconstructed relationships by uniform manifold approximation and projection

88 (UMAP) $^{23}$ to confirm the original structure.

89 


\section{RESULTS}

91 All the dataset used in this paper is summarized in Table 1 and the detailed results are shown in

92 Supplementary Figures and Tables.

Spatial clustering of gene expression data by graph-based SOM with gene set optimization

94 Inspired by Kohonen's self-organizing-map learning theory ${ }^{19}$, here we extended our previously

95 developed method, SPRESSO ${ }^{15}$, to graph-based networks, which is theoretically applicable to

96 cell-to-cell relationships of any types of topological structures of tissues or organs. The basic

97 algorithm of spatial clustering of cells is a combinatorial optimization to find best gene sets to

reproduce known topological network structures of learning objects, or gene vectors of cells. Since

99 each node on the topological network may contain multiple such gene vector objects, given an original topological network, we can calculate the reproducibility of the structure by counting correct and incorrect network edges, either connected or unconnected, after learning process. We also used the Markov-Chain-Monte-Carlo (MCMC) to optimize the best gene sets to give a maximum reproducibility of a topology of structure. We have also tuned the SOM learning process by introducing stochastically learning (i.e., stochastic-SOM ${ }^{15}$ ) that allows learning efficiency in later phase where the extent of learning ability usually decreases monotonously. We refer to this method as eSPRESSO (enhanced-SPRESSO) in this paper. 
108 topological structures are too complicated to be handled and reproduced by our previous SPRESSO,

109 by generating graph structure of 7 domains (Ect1, Ect2, Ect3, PS, MA, MP, E1-E2-E3) with

110 domain-to-domain contact relationships (Fig.1a). We optimized the gene set to maximize the

111 reproducibility of the original graph structure by 1,000 steps of MCMC calculations. To enhance the

112 efficient optimization and stable reproducibility, we performed parallel tempering ${ }^{24}$ based on 8

113 parallel MCMC processes. The resulted clustering is evaluated with the original structure by simply

114 counting the existing edges between two gene vector objects, or cells. We used gene expression data

115 from 213 sections and the reproduced accuracy was $86.3 \%$ after the MCMC optimization and the

116 domain contact map (FIg.1b) of samples for original (lower triangle) and reconstructed (upper

117 triangle) structures (Fig.1c) are shown. A heatmap of gene expression data for optimized SDGs

118 (Fig.1d) is also shown. The final clustering using SDGs is drawn by UMAP (Fig.1e).

120 Circular and hierarchical structure reconstruction by eSPRESSO

121 To evaluate the performance of eSPRESSO, we applied the method to circular and hierarchical

122 structures of tissues. The development of mouse heart initiates with an expansion of a blood vessel,

123 forming complex structure consisting of arteries and veins in later phase. We used scRNA-seq data

124 from mouse embryonic heart structure (E9.25) which represents pseudo-circular structure of seven

125 domains, of which five are projecting from the anterior and posterior second heart fields (AHF and 
126 pSHF) (Fig.2a). When we down-sampled 10 cells for each domain from the initial 3911 cells and

127 optimized the gene set by stochastic-SOM with MCMC to reproduce the original structure, we

128 successfully reconstructed the topology of cellular locations with extremely high accuracy of $96.8 \%$

129 (Fig.2b). The number of final SDGs is 18 which contains many known important genes such as

130 Hand2, Gata4, etc. ${ }^{25}$, in mouse heart development. The visual projection by UMAP clustering of all

1313331 cells using the SDGs show a circular representation of tissue domains which is quite consistent

132 of original structure (Fig.2a). Then we further tried to reconstruct mouse liver structure where a nine layers of tissues

134 from a concentric circle are observed ${ }^{10}$ (Fig.2c). The eSPRESSO correctly reconstructed the 135 relationships of 90 cells, which are randomly selected by down-sampling 10 cells from each of 9

136 domains, with $84.6 \%$ accuracy. The resulted 18 SDGs represent many known liver-specific genes

137 such as cytochrome P450 (Cypla2 and Cyp2f2) and hepatic nuclear factor 4 alpha (Hnf4a) ${ }^{27}$ (Fig.2d).

138 The visual projection by UMAP clustering of all 1415 cells using the 18 SDGs indicate that there are

139 clear layer structure observed although it does not reproduce the circular structure due to the

140 limitation of UMAP clustering that are difficult to generate circular layer topology (Fig.2c).

\section{Developmental analysis of human pancreas by eSPRESSO}

143 One of the powerful characteristics of eSPRESSO may be demonstrated by analysis of organs during 
144 developmental process. We used scRNA-seq data from human developmental pancreas from stage 4,

1455 and 6 data $^{28}$, and reconstructed the structure. The accuracies are high for all the stages such as

146 96-100\%. The numbers of resulted SDGs are 9, 22, and 18, containing pancreas-specific genes such

147 as insulin (INS) and insulin gene enhancer protein ISL-1 (ISL1) ${ }^{29}$ (Supplementary Table1).

148 Interestingly, although we did not input information of endocrine and non-endocrine separation, the

149 UMAP plots of all the thousands of cells using the SDGs indicate that developmental events such as

150 alpha-cell differentiation in Langerhans islets and exocrine cell detachment from endocrine cells are

151 observed (Fig.3a-c).

152 In the stage 4 to 6 reconstructions, the original scRNA-seq data is focusing on endocrine

153 cells; therefore, we obtained endocrine-related genes during stages. GSEA analysis also represent

154 that the developmental changes of SDGs, indicating that more polarization between endo- and

155 exocrine cells seemed to proceed.

157 Application to virtual human gene knockout experiment

158 One of the most useful usages of eSPRESSO may be to perform virtual in-silico human gene

159 knockout experiments. As an example of organs, we used scRNA-seq data from human

160 developmental heart ${ }^{30}$. We first successfully reconstructed the 8 domain structure with accuracy of

$16176.2 \%$ for postconceptional weeks (PCWs) 4.5-5 human embryonic heart (Fig.4a). We obtained \#\# 
162 SDGs as the optimized result (Supplementary Table1). Then we performed single gene deletions

163 among the SDGs to see if any structural changes are observed (Fig.4b). Among the 37 SDGs, most

164 effective genes that dramatically decreases accuracies of reconstructions are found (Supplementary

165 Table2). The structural change for gene $\mathrm{X}$ deletion is shown in Fig.4c. The gene $\mathrm{X}$ is the orthologous

166 gene in mouse that is homozygous lethal. The decreases of accuracies are also shown

167 (Supplementary Table2). All other structural changes are shown in Supplementary Figure 1. We also

168 performed two gene deletions (Supplementary Table3).

169 We also performed similar analyses for scRNA-seq data from PCW 6.5 and 9 human

170 hearts. The most effective genes that decreases accuracies of reconstructions are shown in

171 Supplementary Table 4.

172 


\section{Discussion}

174 In this paper, we developed a computational approach that combines the SOM

175 clustering under the topological constraints and gene set optimization by MCMC. The

176 reproducibility of tissue topology is sufficiently high and this method may innovatively

177 add more information on the existing clustering method such as helping to find spatially

178 distributed discriminator genes (SDGs) and inference of developmental architecture of

179 tissue domains. It is also useful to investigate the collapses of spatial clustering by

180 in-silico virtual gene knockouts although it is not a real knock-out experiments.

181 Recently, alternative direct experimental approaches such as highly-multiplexed

182 FISH or direct spatial transcriptome have been proposed. However, the primal purpose

183 of these methods is to reconstruct cellular locations with gene expression data. Although

184 they can contribute to detect differentially expressed genes among distinct tissue

185 domains, it does not explicitly consider the tissue topologies and thus, it may lack to

186 detect spatially contributing genes in terms of neighboring tissue networks. Our method

187 may be able to complement such a neighboring tissue information and it is also

188 applicable to the results of such data to detect globally expressed genes that contributes

189 to domain networks in spatial distributing manner

190 Our methods may have benefits in analysis of organ development. Many genes 
191 show homozygous lethality in development. Although many evidences based on animal

192 studies indicate that there are candidates of those lethal genes in human, it is not

193 possible to reproduce lethality in human system due to ethical reasons. The human

194 organoids derived from iPS or ES cells may provide an alternative approach to

195 reproduce gene knockout organs. However, abnormalities are often stochastic and

196 affected by other internal or external factors and it sometimes requires a large number of

197 repeat experiments to reproduce the phenomena ${ }^{31}$. Contrastingly, our in-silico virtual

198 knockout method does not require any cumbersome protocols and may be able to

199 reproduce the same results in many times.

The SOM learning is a renowned method in the field of clustering, first

201 proposed by Kohonen and colleagues ${ }^{19}$. They have already introduced graph-network

202 topology for SOM clustering but there existed not many examples to solve in their

203 days $^{18}$. The original SOM learning decreases learning regions and efficiency

204 monotonously, which causes clustering problems in the later phase and we found that

205 efficient scheduling for learning was necessary for practical application to the current

206 single-cell transcriptome datasets. Thus, we introduced the stochastic-SOM learning

207 which has a characteristic similar to that of Gibbs-sampling although the sampling

208 probability is totally equal. This improvement dramatically enhanced the clustering 
209 ability and thus enabled to implement this study.

210 Finally, we state that our current algorithms and analysis pipeline describe here

211 is still primitive and many modifications are necessary. The extraction of initial gene

212 candidates are solely dependent on Boruta package that detects feature genes by random

213 forest generation and may miss important genes that overlaps categories in the distant

214 branches or leaves. The parallel tempering MCMC is also a suboptimal approach,

215 although it enhances the combinatorial optimization to allow quick convergence to the

216 global optimum, which sometimes fail to find the best gene set to reproduce the

217 topology by SOM clustering. We expect that more efficient and fine-grained algorithms

218 to find global optimum in decent time may replace modules in our pipeline in future. 


\section{Methods}

221 Methods and any associated references are available in the online version of the paper.

222 Note: Any Supplementary Information and Source Data files are available in the online

223 version of the paper.

\section{Code and data availability}

226 The proposed methods including the feature gene selection, the 3D reconstruction using

227 stochastic-SOM clustering, and visualization, are implemented in R and Python and are available at

228 https://github.com/tmorikuicr/espresso. Also all the gene expression data used in this study is

229 included together. 


\section{$230 \quad$ References}

231 1. Rossi, M. A. et al. Obesity remodels activity and transcriptional state of a lateral 232 hypothalamic brake on feeding. Science (80-. ). 364, 1271-1274 (2019).

233 2. Jackson, H. W. et al. The single-cell pathology landscape of breast cancer. $234 \quad$ Nature 578, 615-620 (2020).

235 3. Litviňuková, M. et al. Cells of the adult human heart. Nature Published, (2020).

236 4. Battich, N., Stoeger, T. \& Pelkmans, L. Image-based transcriptomics in thousands of single human cells at single-molecule resolution. Nat. Methods

5. Lubeck, E. \& Cai, L. Single-cell systems biology by super-resolution imaging and combinatorial labeling. Nat. Methods (2012) doi:10.1038/nmeth.2069.

6. Chen, K. H., Boettiger, A. N., Moffitt, J. R., Wang, S. \& Zhuang, X. Spatially resolved, highly multiplexed RNA profiling in single cells. Science (80-. ).

9. Achim, K. et al. High-throughput spatial mapping of single-cell RNA-seq data to (2015) doi:10.1126/science.aaa6090.

7. Ståhl, P. L. et al. Visualization and analysis of gene expression in tissue sections by spatial transcriptomics. Science (2016) doi:10.1126/science.aaf2403.

8. Satija, R., Farrell, J. A., Gennert, D., Schier, A. F. \& Regev, A. Spatial reconstruction of single-cell gene expression data. Nat. Biotechnol. (2015) doi:10.1038/nbt.3192.

10. Halpern, K. B. et al. Single-cell spatial reconstruction reveals global division of labour in the mammalian liver. Nature (2017) doi:10.1038/nature21065.

11. Durruthy-Durruthy, R. et al. Reconstruction of the mouse otocyst and early 
12. Durruthy-Durruthy, R., Gottlieb, A. \& Heller, S. 3D computational reconstruction of tissues with hollow spherical morphologies using single-cell gene expression data. Nat. Protoc. (2015) doi:10.1038/nprot.2015.022.

13. Durruthy-Durruthy, J. et al. Spatiotemporal Reconstruction of the Human Blastocyst by Single-Cell Gene-Expression Analysis Informs Induction of Naive Pluripotency. Dev. Cell (2016) doi:10.1016/j.devcel.2016.06.014.

14. Li, J. et al. Systematic Reconstruction of Molecular Cascades Regulating GP Development Using Single-Cell RNA-Seq. Cell Rep. (2016) doi:10.1016/j.celrep.2016.04.043.

15. Mori, T., Takaoka, H., Yamane, J., Alev, C. \& Fujibuchi, W. Novel computational model of gastrula morphogenesis to identify spatial discriminator genes by self-organizing map (SOM) clustering. Sci. Rep. (2019) doi:10.1038/s41598-019-49031-1.

16. Ashburner, M. et al. Gene ontology: Tool for the unification of biology. Nature Genetics (2000) doi:10.1038/75556.

17. Carbon, S. et al. Expansion of the gene ontology knowledgebase and resources: The gene ontology consortium. Nucleic Acids Res. (2017) doi:10.1093/nar/gkw1108.

18. Kangas, J. A., Kohonen, T. K. \& Laaksonen, J. T. Variants of Self-Organizing Maps. IEEE Trans. Neural Networks (1990) doi:10.1109/72.80208.

19. Kohonen, T. Self-organized formation of topologically correct feature maps. Biol. Cybern. (1982) doi:10.1007/BF00337288.

20. von der Malsburg, C. Self-organization of orientation sensitive cells in the striate cortex. Kybernetik (1973) doi:10.1007/BF00288907.

21. Turing, A. M. The chemical basis of morphogenesis. Philos. Trans. R. Soc. Lond. B. Biol. Sci. 237, 37-72 (1952).

22. Li, X., Gasteiger, J. \& Zupan, J. On the topology distortion in self-organizing 
feature maps. Biol. Cybern. (1993) doi:10.1007/BF00200832.

23. McInnes, L., Healy, J. \& Melville, J. UMAP: Uniform manifold approximation and projection for dimension reduction. arXiv (2018).

24. Swendsen, R. H. \& Wang, J. S. Replica Monte Carlo simulation of spin-glasses. Phys. Rev. Lett. (1986) doi:10.1103/PhysRevLett.57.2607.

25. Duan, J. et al. Rational Reprogramming of Cellular States by Combinatorial Perturbation. Cell Rep. (2019) doi:10.1016/j.celrep.2019.05.079.

26. Subramanian, A. et al. Gene set enrichment analysis: A knowledge-based approach for interpreting genome-wide expression profiles. Proc. Natl. Acad. Sci. U. S. A. (2005) doi:10.1073/pnas.0506580102.

27. Ben-Moshe, S. et al. Spatial sorting enables comprehensive characterization of liver zonation. Nat. Metab. (2019) doi:10.1038/s42255-019-0109-9.

28. Veres, A. et al. Charting cellular identity during human in vitro $\beta$-cell differentiation. Nature (2019) doi:10.1038/s41586-019-1168-5.

29. Zhang, D. et al. Highly efficient differentiation of human ES cells and iPS cells into mature pancreatic insulin-producing cells. Cell Res. (2009) doi:10.1038/cr.2009.28.

30. Asp, M. et al. A Spatiotemporal Organ-Wide Gene Expression and Cell Atlas of the Developing Human Heart. Cell (2019) doi:10.1016/j.cell.2019.11.025.

31. Bhaduri, A. et al. Cell stress in cortical organoids impairs molecular subtype specification. Nature (2020) doi:10.1038/s41586-020-1962-0. 


\section{Acknowledgements}

306 The authors deeply appreciate Dr. Peter Karagiannis for kindly reviewing the manuscript. This work

307 was partially supported by the Core Center for iPS Cell Research, Research Center Network for

308 Realization of Regenerative Medicine, Japan Agency for Medical Research and Development

309 (16bm0104001h0004), and the iPS Cell Research Fund.

\section{Author contributions}

312 W.F. conceptualized and designed the study. T.M. implemented the software. T.M., K.L. and T.T.

313 performed the computational experiments. J.Yamane., C.A., K.O., and J.Yamashita. performed the

314 data interpretation from a biological point of view. T.M. and W.F. wrote the manuscript. All authors

315 have read and approved the final manuscript.

\section{Competing Interests}

318 The authors declare no competing interests. 


\section{Figure Legends}

320 Figure 1. Overview of the 3D reconstruction method of mouse embryo using stochastic-SOM

321 clustering with graph-network topology.

322 Figure 2. Examples of 3D reconstructions: a,b) mouse developmental heart and c,d) mouse

323 liver.

324 Figure 3. Example of 3D reconstructions during pancreas development.

325 Figure 4. Example of virtual knockout analysis by eSPRESSO in human developmental heart. 


\section{Data Name}

Mouse embryo (E7.0)

Peng, Guangdun, et al. "Spatial transcriptome for the molecular annotation of

lineage fates and cell identity in mid-gastrula mouse embryo."

Developmental cell 36.6 (2016): 681-697.

Peng, Guangdun, et al. "Molecular architecture of lineage allocation and

Mouse embryo (E5.5-7.5) tissue organization in early mouse embryo." Nature 572.7770 (2019): 528-

Mouse brain (ALM)

Mouse brain (VISp)

Mouse heart (E7.75)

Mouse heart (E8.25)

Mouse heart (E9.25)

Mouse liver

Human pancreas (S4)

Human pancreas (S5)

Human pancreas (S6)

Human heart (4.5-5PCW)

Human heart (6.5PCW)

Human heart (9PCW)
Tasic, Bosiljka, et al. "Shared and distinct transcriptomic cell types across neocortical areas." Nature 563.7729 (2018): 72-78.

de Soysa, T. Yvanka, et al. "Single-cell analysis of cardiogenesis reveals basis for organ-level developmental defects." Nature 572.7767 (2019): 120-

Halpern, Keren Bahar, et al. "Single-cell spatial reconstruction reveals global division of labour in the mammalian liver." Nature 542.7641 (2017): 352-356.

Veres, Adrian, et al. "Charting cellular identity during human in vitro $\beta$-cell differentiation." Nature 569.7756 (2019): 368-373.

Asp, Michaela, et al. "A spatiotemporal organ-wide gene expression and cell atlas of the developing human heart." Cell 179.7 (2019): 1647-1660.
\#Domain

\#Cells

$4 \quad 41$ (sections)

7213 (sections)

3809

7049

1259

3331

3911

$9 \quad 1415$

$5 \quad 5273$

$5 \quad 3925$

8238 (spots)

1515 (spots

8 1358 (spots)
\#Initial genes

\#SDGs Max Accuracy

ARI

185

18

$100.0 \%$

17

$86.3 \%$

0.79731

$100.0 \%$

$100.0 \%$

$100.0 \%$

$97.5 \%$

$84.6 \%$

$95.9 \%$

$97.6 \%$

$95.5 \%$

$76.2 \% \quad 0.5314551$

$70.6 \% \quad 0.3526241$

$72.5 \% \quad 0.3513529$

Table 1. Datasets and performances of 3D structure reconstructions (score $=$ accuracy + ARI) 
(a)

\section{Graph-SOM}
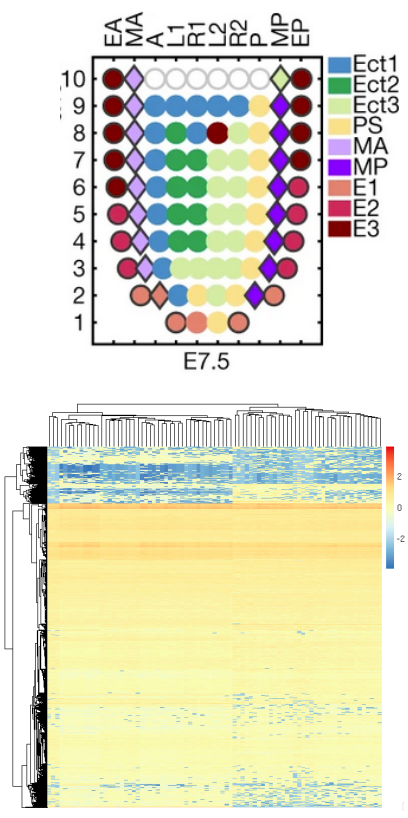

Feature genes
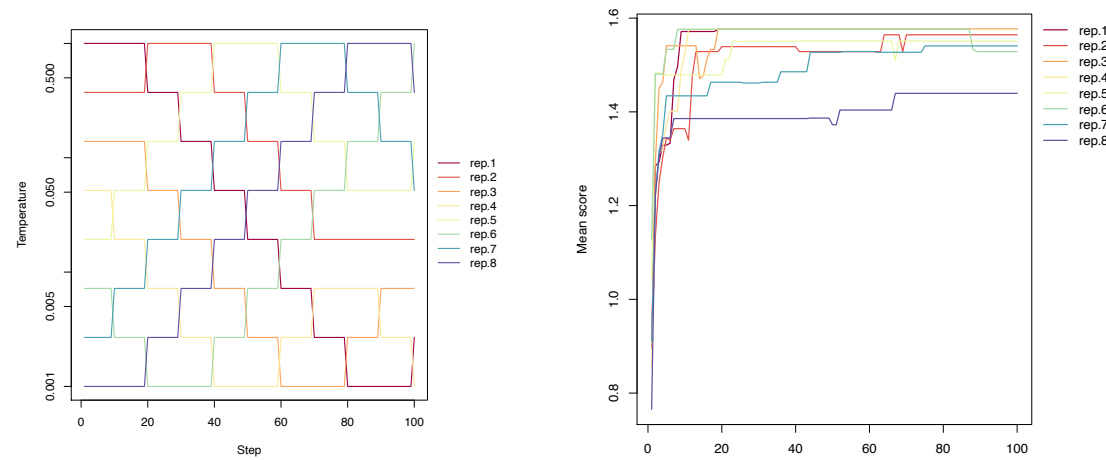

Combinatorial Optimization of Genes (Markov Chain Monte Carlo)

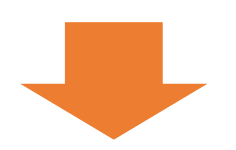

(b)

(c)



(d)

(e)

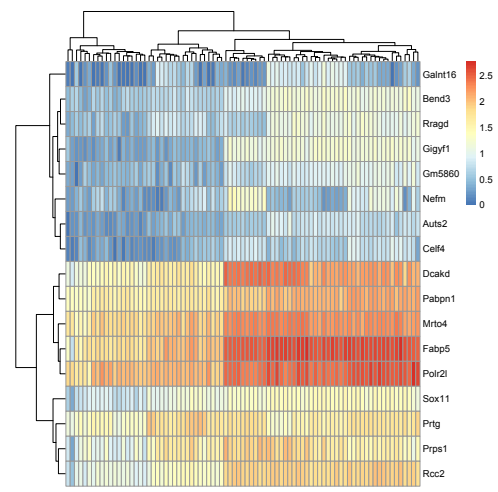

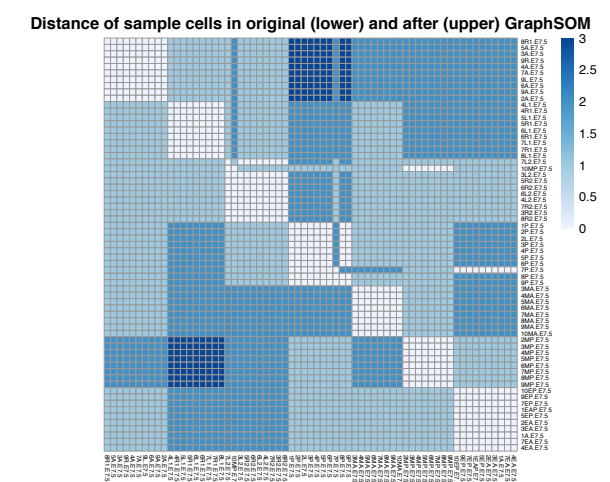

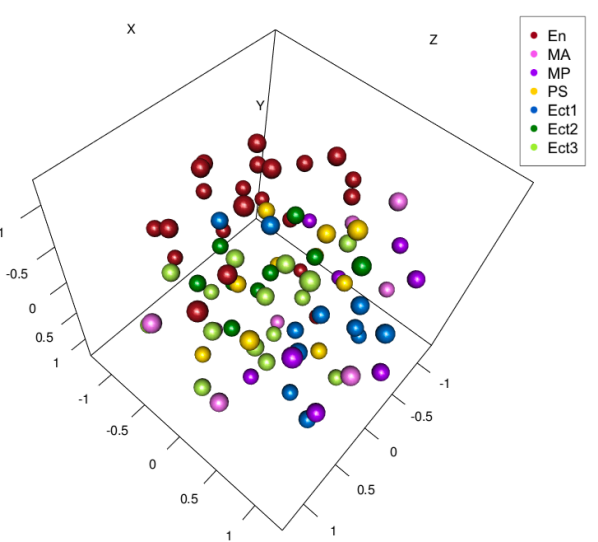




\section{Figure 2}

(a)

Mouse developmental heart

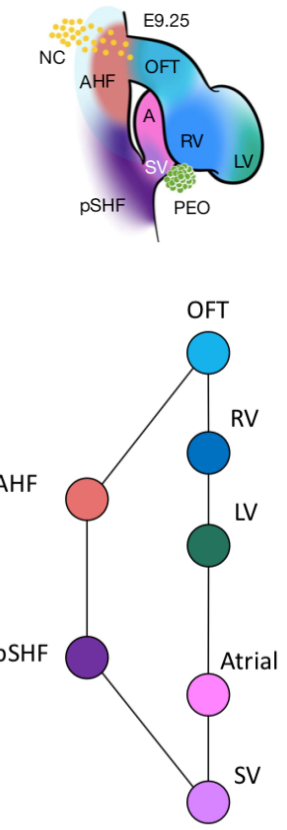

(b)

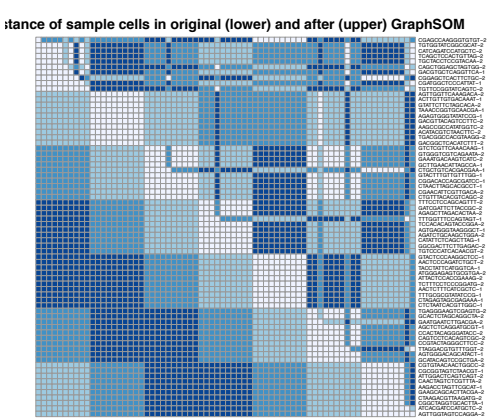

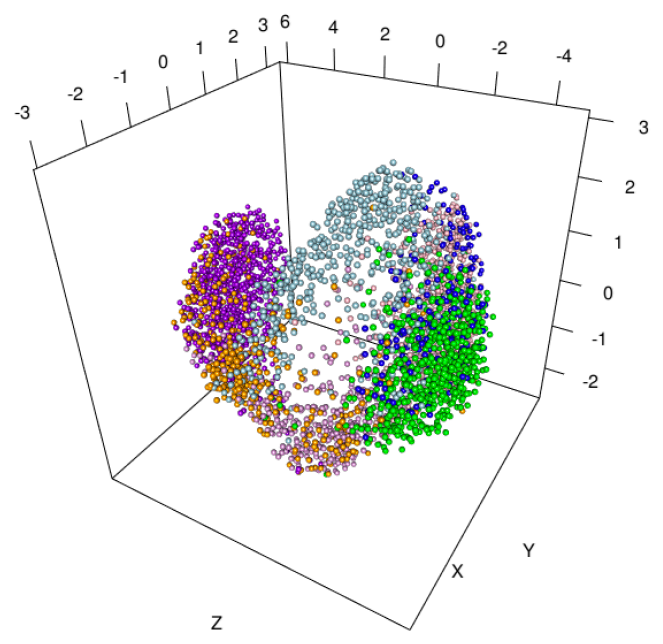

(d) (c)

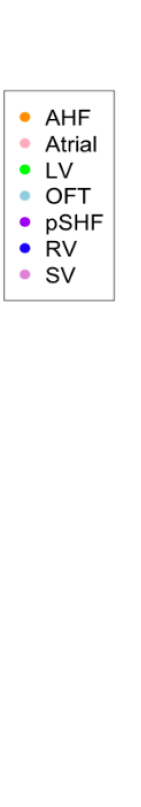

Mouse liver

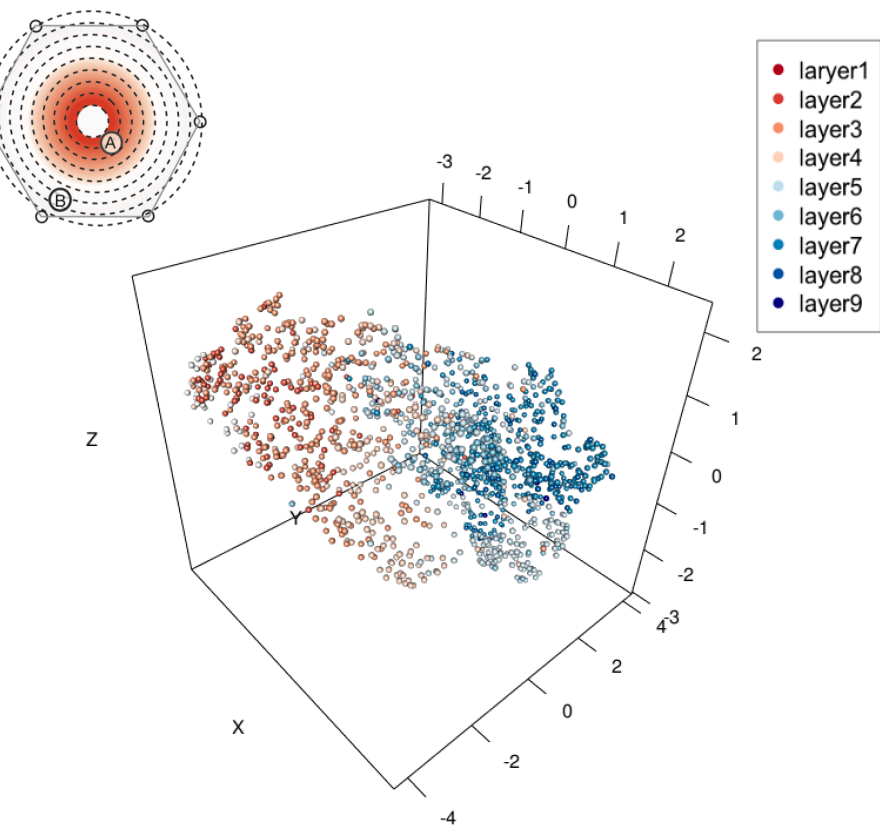

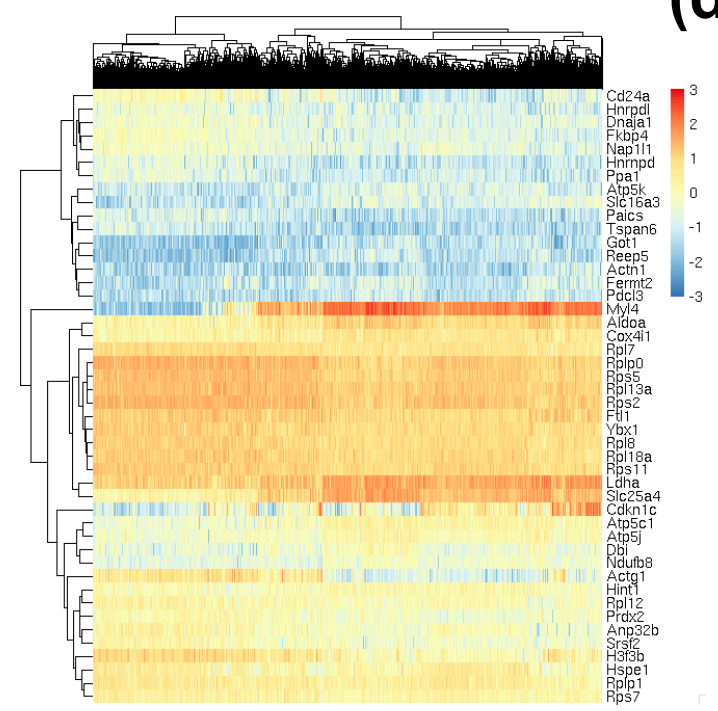






\section{Human developmental pancreas}

(stage 4 to 6)

(a)

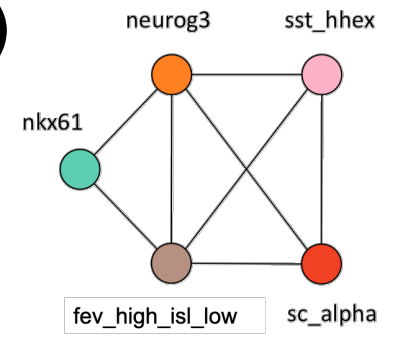

- fev_high_isl_low$$
\text { - neurog3 }
$$$$
\text { - nkx61 }
$$$$
\text { - sc_alpha }
$$
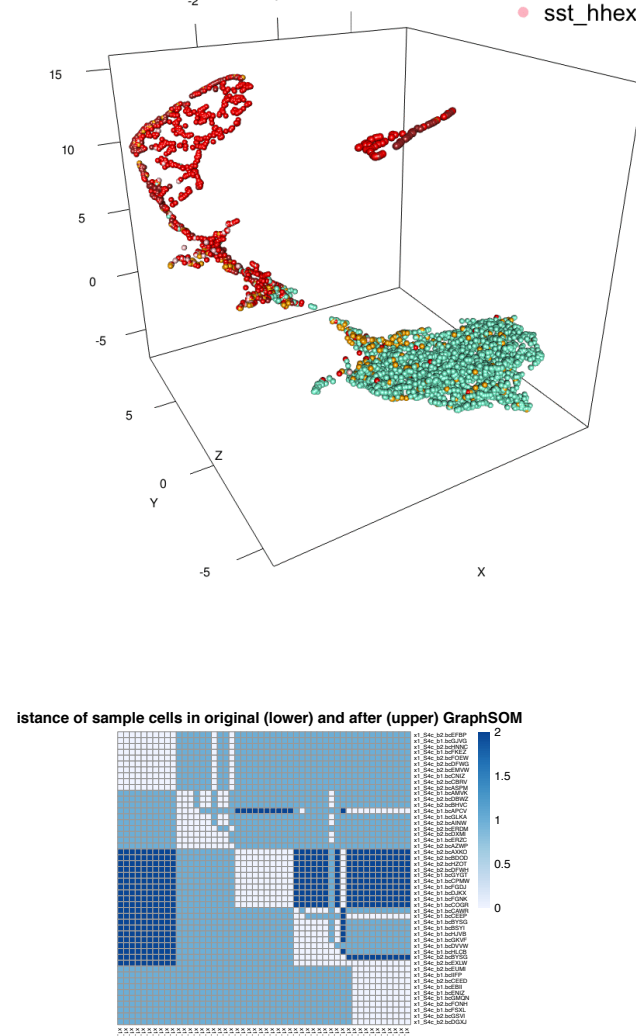

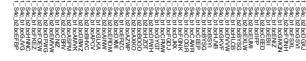

(b)

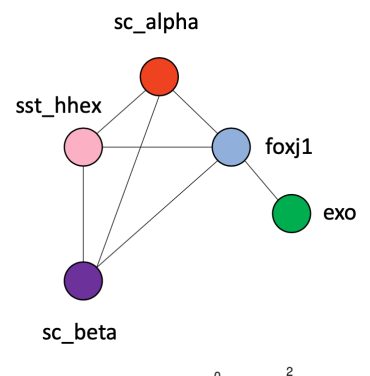

- exo

- foxj1

- sc_beta

- sst_hhe:

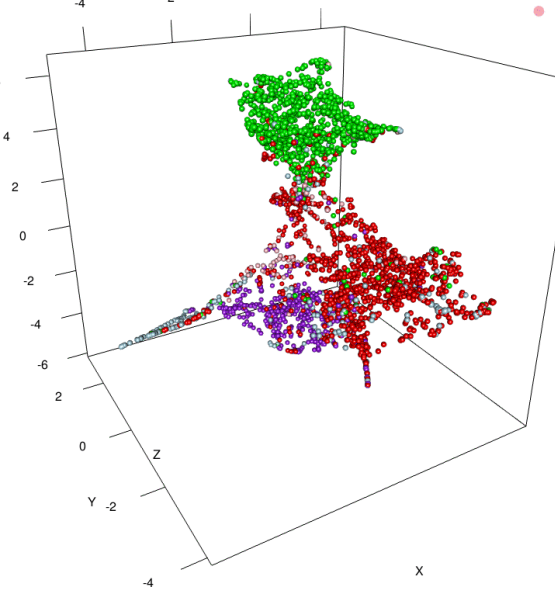

istance of sample cells in original (lower) and after (upper) Graphsom

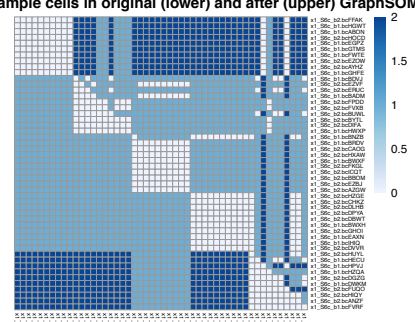



(c)

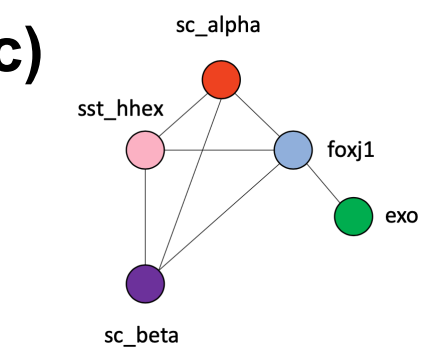

- exo

- foxj1

- sc_beta

sst_hhex

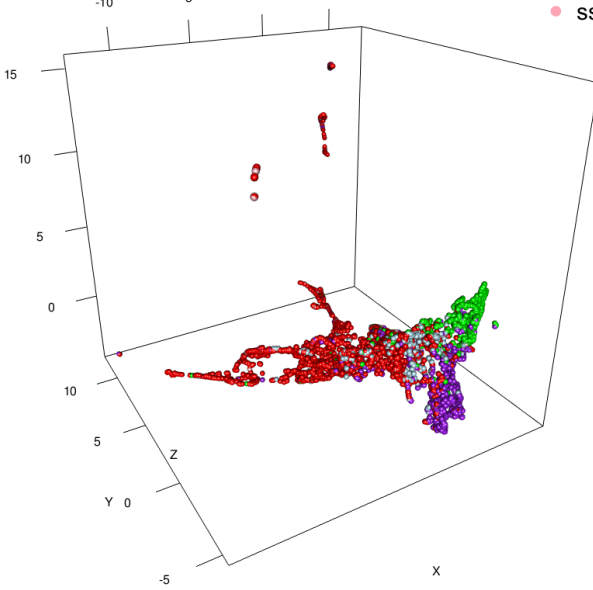

istance of sample cells in original (lower) and after (upper) Graphso

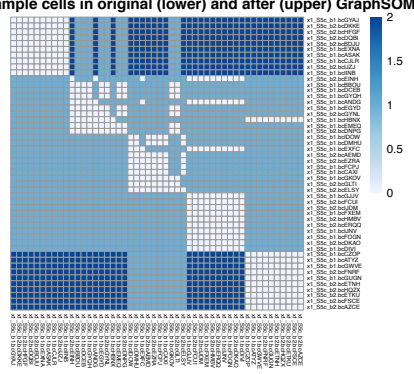




\section{Human developmental heart}

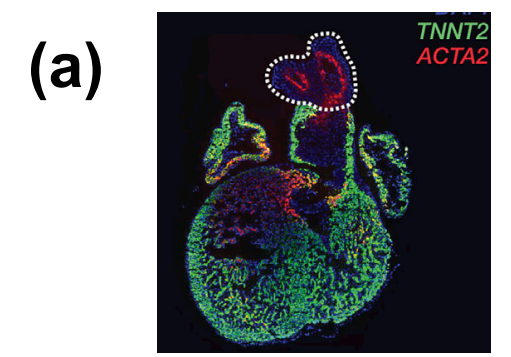

CVM

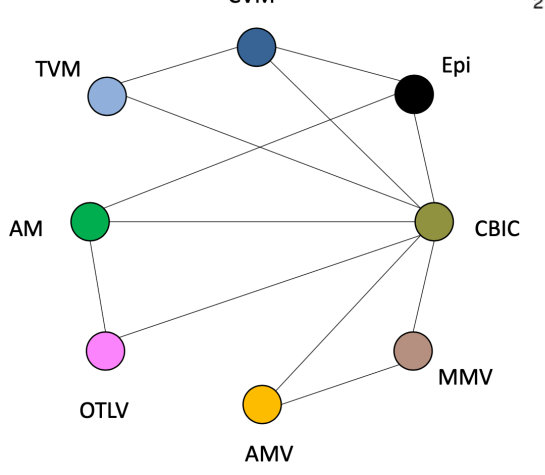



(b) VKO analysis

(c) Before -> After



